\section{Cahiers d'ethnomusicologie}

Anciennement Cahiers de musiques traditionnelles

$13 \mid 2001$

Métissages

\title{
Musiques du monde arabe à Alep
}

Compte rendu du troisième congrès du Groupe d'étude du Conseil international de la musique traditionnelle sur les musiques du monde arabe. Alep, Syrie, 28 avril au $1^{\text {er }}$ mai 2000.

\section{Veronica Doubleday}

Traducteur : Ramèche Goharian

\section{OpenEdition}

\section{Journals}

Édition électronique

URL : http://journals.openedition.org/ethnomusicologie/783

ISSN : 2235-7688

Éditeur

ADEM - Ateliers d'ethnomusicologie

Édition imprimée

Date de publication : 1 janvier 2001

Pagination : 267-271

ISBN : 2-8257-0723-6

ISSN : 1662-372X

Référence électronique

Veronica Doubleday, « Musiques du monde arabe à Alep », Cahiers d'ethnomusicologie [En ligne], 13 | 2001, mis en ligne le 09 janvier 2012, consulté le 05 mai 2019. URL : http://

journals.openedition.org/ethnomusicologie/783

Ce document a été généré automatiquement le 5 mai 2019.

Tous droits réservés 


\title{
Musiques du monde arabe à Alep
}

\author{
Compte rendu du troisième congrès du Groupe d'étude du Conseil \\ international de la musique traditionnelle sur les musiques du monde \\ arabe. Alep, Syrie, 28 avril au $1^{\mathrm{er}}$ mai 2000.
}

\author{
Veronica Doubleday \\ Traduction : Ramèche Goharian
}

1 Peu de conférences d'ethnomusicologie offrent aux participants l'occasion d'écouter de la musique autrement que sur un plan purement analytique. Mais Scheherazade Hassan, présidente du groupe d'étude du CIMT, proposa une approche différente en programmant dans le cadre du congrès d'Alep, qui fut une grande réussite, un large éventail d'événements musicaux. Le choix pour cette rencontre d'Alep, centre important de musique et de culture arabes, était particulièrement approprié. Cette ville ancienne possède une citadelle célèbre, un grand musée et douze kilomètres de marché couvert qui sont probablement les suq les mieux préservés de tout le Moyen-Orient. Nous disposions de temps libre pour visiter ces sites et, à deux reprises, nous dînâmes dans des restaurants aménagés dans de ravissantes maisons ornées de fontaines de marbre et de plafonds en bois peint ou gravé, nous invitant à établir des rapprochements entre la musique et les arts visuels de la Syrie.

2 Des musiciens et intellectuels alepins contribuèrent à enrichir notre colloque en se joignant à notre groupe d'une vingtaine de participants, en jouant de la musique et animant les débats. Parmi eux, Nuri Iskander, directeur de l'Institut de musique d'Alep, joua un rôle essentiel dans l'organisation des concerts. Notre hôte principal, un charmant prêtre syriaque catholique, le père Emile Assouad, offrit généreusement de nous réunir dans son église et couvent de Saint Assia, datant $\mathrm{du}_{\mathrm{xv}}^{\mathrm{e}}$ siècle, situés dans un vieux quartier chrétien de la ville. A l'abri du bruit et du trafic, l'atmosphère calme et harmonieuse de ce lieu incitait à la discussion et à l'appréciation de la musique.

3 Le congrès, réuni sur invitation de l'Institut français des études arabes de Damas, (IFEAD) regroupait des participants originaires de différentes parties du monde arabe (Syrie, Liban, Jordanie, Yémen, Bahrein et Irak), d'Europe et des Etats-Unis. Les exposés portèrent sur trois sujets : la musique arabe à l'époque ottomane, la musique bédouine et 
tribale dans le monde arabe, ainsi que la terminologie et les concepts relatifs aux musiques du monde arabe. Le colloque se déroulant en trois langues : français, arabe et anglais, le temps dévolu à la traduction empiéta quelque peu sur celui réservé aux discussions formelles; cependant la dimension réduite de l'assemblée permit de fructueux échanges d'idées.

Dominique Mallet, directeur de l'IFEAD, ouvrit le colloque par un discours plein d'esprit avant de présenter un exposé inaugural très érudit sur la musique, la poésie et la politique dans l'œuvre $\mathrm{du}$ théoricien $\mathrm{du} \mathrm{x}^{\mathrm{e}}$ siècle, al-Farabi. La première session, consacrée à la musique bédouine dans le monde contemporain, réunissait trois exposés. Abdul-Hamid Hamam de Jordanie présenta des extraits de vidéo tournés lors d'une cérémonie de circoncision pour illustrer les chants de célébration antiphoniques féminins (tarwida). Sa'di al-Hadîthi, chanteur et folkloriste d'Irak, proposa des exemples musicaux des Bédouins du désert irakien en insistant particulièrement sur les genres hida' et hjeini. Le savant syrien Mahmud Ismail parla de la danse dahha des Bédouins des régions syriennes de l'Euphrate, en mettant l'accent sur les analyses mélodiques et rythmiques.

5 Le colloque était soigneusement programmé pour nous permettre d'assister aux cérémonies musulmanes et chrétiennes comportant des chants et de la musique instrumentale. C'est ainsi que notre première après-midi fut consacrée à la musique religieuse dans son contexte. Tout d'abord, nous eûmes le privilège d'assister à la cérémonie hebdomadaire de dhikr de vendredi après-midi du Tekkie al-Badenjki, un remarquable rituel soufi où l'invocation des noms de Dieu est accompagnée de chants et de percussions selon les traditions élaborées dans les ordres Qaderi et Rifaï. Les hommes étaient autorisés à pénétrer dans la salle de cérémonie alors que les femmes regardaient et écoutaient à partir d'un podium dressé dans la cour. Après quelques heures de repos, nous fûmes invités à prendre place à l'avant de la grande église syriaque orthodoxe d'Alep, qui était comble, pour assister au service de nuit du Vendredi Saint de la Pâque dans la tradition de l'Edesse-Urfa. Aux chants liturgiques des hommes, interprétés par plusieurs prêtres dont le père Emile Assouad, s'ajoutèrent des sections de chœurs antiphoniques masculins, des chants solo d'une femme accompagnés d'un synthétiseur situé sur la galerie supérieure à l'arrière de l'église, et de rituels accomplis autour du cercueil fleuri de Jésus-Christ. Après le service, l'Archevêque Gregorius Ibrahim nous offrit une réception spéciale où il nous fit part de son intérêt personnel pour la musicologie et souligna l'importance des travaux d'un de nos participants, le père libanais Elie Kesrouani, qui avait enregistré et transcrit le cycle liturgique complet d'une année de l'Eglise syriaque orthodoxe, sujet de sa thèse de doctorat à la Sorbonne.

6 La deuxième session fut dédiée à la musique et aux chants tribaux relatifs à certains aspects de la culture bédouine. Trois des quatre exposés furent présentés par des spécialistes venus de Paris. Monique Brandily décrivit la cérémonie nuptiale de quatre jours qui se déroule au Fezzan, en Libye, et comporte des chants et de la musique instrumentale pour tambours et clarinette double. Miriam Rovsing Olsen mentionna les rapports formels qui existent entre les cycles musicaux et agricoles/saisonniers chez les Berbères des montagnes du Haut-Atlas, au Maroc, en mettant l'accent sur la danse collective ahwash qui conclut la moisson de l'orge. Habib Yammine analysa un art exclusivement masculin, la danse bar'a des régions montagneuses du Yémen, en se concentrant sur sa structure rythmique et son importance en tant qu'indicateur de l'identité tribale. Le professeur Jürgen Elsner de Berlin présenta des données modales de la tradition nomade Aiyai du désert saharien d'Algérie, en s'intéressant à la terminologie 
locale relative au mouvement mélodique; il utilisa des exemples musicaux et des transcriptions schématiques pour démontrer l'importance de la quarte et de la quinte comme intervalles constituants.

7 La troisième session mit en évidence les importantes traditions classiques et religieuses d'Alep. Ustad Muhammad Qaderi Dalal, grand joueur de 'ud et intellectuel de cette ville fit un exposé détaillé sur la musique religieuse islamique à Alep, illustré de beaux extraits chantés par lui-même ainsi que par d'éminents muezzins et chanteurs de la ville, dont le révéré muezzin et compositeur Sabri al-Mudallal, et l'expressif et talentueux chanteur Omar Sermini. Une partie de l'exposé fut consacrée à l'analyse du dhikr soufi alepin.

Ustad Dalal dirigea ensuite un groupe de musiciens dans un excellent concert de musique classique locale; il y jouait lui-même du 'ud, les autres instruments étant le qanun, le nay, le violon, le riqq et le darbukka. Nous entendîmes également du qanun joué avec virtuosité par Ustad Hasan al-Tannari. La musique continua après le dîner dans le jardin de Saint Assia avec ses arbres en fleurs et sa haute galerie ouverte, l'iwan. Sa'di al-Hadîthi donna le ton avec son solo de chants bédouins plaintifs et émouvants, suivis d'un superbe récital de musique classique arabe offert par le chanteur Omar Sermini, accompagné des musiciens précités et de Habib Yammine au riqq. L'intensité émotive que dégage la poésie chantée dans la musique arabe était quasiment palpable.

9 Lors de sa réunion de 1996 à Oxford, le groupe d'étude avait voté pour un projet de préparation de dictionnaires terminologiques de musique arabe. Scheherazade Hassan et Leo Plenckers d'Amsterdam avaient déjà passé plusieurs mois à étudier ce projet. Notre quatrième session fut consacrée à des communications sur la terminologie et la cinquième laissa un peu de temps à Scheherazade et Leo pour présenter leurs exposés, suivis d'un débat.

10 Au cours de la quatrième session, Jonathan Shannon de New York discuta de certains concepts relatifs au dhikr alepin en les rapprochant du wasla exécuté dans cette ville. Vinrent ensuite deux communications sur le Yémen : celle de Jean Lambert de Paris sur les rapports entre la terminologie relative aux formes mélodiques et celle servant à désigner les parties du 'ud yéménite, celle de Jabar Ali Ahmad, directeur du Centre de musique traditionnelle de Sanaa, sur la riche terminologie rythmique au Yémen. Mubarak 'amr al-Ammari de Bahrein présenta des documents sur le genre sowt dans le Golfe et le Yémen, et Leo Plenckers fit quelques remarques analytiques préliminaires sur le mawwal syrien, un genre important, mais qui n'a pas toujours retenu l'attention qu'il méritait.

11 Scheherazade Hassan ouvrit la cinquième session en examinant la littérature terminologique de la musique arabe et en soulignant la nécessité de publier des documents mis à jour tenant compte des recherches menées sur le terrain dans tout le monde arabe. Elle proposa de compiler dans un premier temps les définitions terminologiques du Groupe d'étude en arabe, quitte à les traduire plus tard dans d'autres langues. Elle suggéra que des dictionnaires séparés soient réalisés pour les régions suivantes : l'Afrique du Nord, l'Egypte, la Syrie, le Liban, la Jordanie et la Palestine, l'Irak, la Péninsule arabique et la région du Golfe, les coordinateurs régionaux gardant la possibilité de recueillir des données pour des dictionnaires individuels. Leo Plenckers fit une esquisse utile des nombreux problèmes techniques soulevés par une telle entreprise et des décisions nécessaires pour sa mise en chantier. La question du financement reste ouverte. 
12 La sixième et dernière session comporta cinq exposés sur la musique arabe à l'époque ottomane. John Morgan O'Connell de Limerick, en Irlande, parla du style arabe dans la musique turque au début du $\mathrm{xx}^{\mathrm{e}}$ siècle, ainsi que des réactions officielles contre le style ottoman. Scheherazade Hassan décrivit la musique et les musiciens en Irak à la fin de la période ottomane, avec l'éclosion du maqam al-'iraqi en tant que tradition locale. Elle souligna l'importance de la musique dans les cercles religieux et montra la place qu'occupait Bagdad comme centre multi-culturel. Gérard Rayissian de Midan, en Syrie, livra des informations importantes sur le rôle joué à l'époque par les musiciens et les compositeurs arméniens, dont les chantres de l'amour, les ashug, dans une vaste aire géographique. Mahmud al-Ajjam de Latakia, en Syrie, présenta des documents historiques sur la musique syrienne à l'époque ottomane. Nous avions la chance d'avoir comme président et conférencier le docteur Saad Allah Agha al-Kalaa, célèbre érudit de l'université de Damas dont le père, Fu'ad Raja $i$, fonda le premier Institut de musique syrienne, à Alep. Le docteur Saad Allah jeta un précieux éclairage sur les rapports historiques qui existent entre le chant arabe et la musique turque depuis le xviie siècle et s'arrêta sur différentes questions soulevées par d'autres exposés.

Les soirs, nous eûmes le plaisir d'entendre deux autres excellents concerts. Dans le premier, un groupe de jeunes gens et de jeunes femmes, Al-Fakharrun, interpréta, sous la direction de Nuri Iskander, des chants syriaques, suivis d'un échange informel d'interprétations solo entre les musiciens alepins et les membres du Groupe d'étude, Mahmud al-Ajjam, Jabar Ali Ahmad et Jean Lambert. Notre dernier concert fut animé par l'Ensemble des traditions musicales alepines, Firqat al-turath al-halebi, placé sous la direction de Ahmed Hamadiyya: accompagné d'un orchestre, un chœur d'hommes y interpréta des chants joyeux agrémentés de nombreuses parties solistes. Dans l'ensemble la musique entendue au cours des concerts ou dans les exemples musicaux illustrant les exposés représentait la face publique de la musique traditionnelle arabe et comportait relativement peu de contributions féminines. Les femmes interprètes apparaissent dans les ensembles syriens modernes, et il est encourageant de constater la présence de femmes parmi les instrumentistes et les enseignants de l'Institut Nuri Iskander d'Alep ; mais les ensembles de musique les plus traditionnels restent essentiellement masculins.

Le colloque fut remarquable à plusieurs égards, ne serait-ce que par son atmosphère paisible de respect mutuel. J'attribue ceci à la tradition d'hospitalité et de tolérance religieuse d'Alep ainsi qu'au pouvoir unificateur de la musique partagée. Les contacts intellectuels fructueux se nouent plus facilement dans de petits cercles, et cette réunion offrait un agréable contraste avec les tractations et autres jeux de coudes effrénés qui sévissent dans certaines conférences académiques, en particulier dans le "grand cirque » des congrès internationaux. Le succès de ce colloque était aussi dû en grande partie à la présence de musiciens locaux qui jouèrent volontiers pour nous, par plaisir et fierté pour leur musique, nous aidant à asseoir nos discussions sur des bases solides.

Sur le plan scientifique, les participants réunirent un grand nombre de documents intéressants provenant de différentes régions $\mathrm{du}$ monde arabe, et le projet de dictionnaires montra à l'évidence la nécessité d'approfondir la recherche sur le terrain et de développer la publication de nombreux autres sujets. Les thèmes choisis par le Groupe d'étude tournaient essentiellement autour de la musique traditionnelle, mais il y eut aussi des discussions informelles très utiles sur la politique et l'impact de la commercialisation sur la musique et les musiciens arabes, surtout dans leurs rapports avec les concerts à Paris. 
Sur le plan intellectuel, j'ai relevé des différences d'approche intéressantes entre les analystes locaux et ceux formés en Occident. Il existe actuellement une mode académique occidentale, spécialement américaine, qui consiste à favoriser la théorie aux dépens de certains aspects de la pratique. L'approche arabe a tendance à se concentrer sur le détail musicologique et ethnographique. Alors qu'ils enracinent leurs idées dans la théorie musicale et rythmique traditionnelle arabe, les chercheurs établis dans les pays arabes n'apprécient pas toujours la tendance occidentale à théoriser, surtout lorsque cela signifie simplifier et rationaliser les arguments dans le but de tirer des conclusions intéressantes, voire brillantes. Les musicologues d'Alep remettent aussi en question la pratique occidentale d'effectuer des transcriptions schématiques pour des raisons analytiques: ils laissent entendre que l'on pourrait facilement invalider les critères d'élimination des détails et qu'il ne faut pas écarter trop rapidement certains aspects de la musique jugés secondaires ou sans importance.

Nous sommes redevables au docteur Dominique Mallet et à l'IFEAD d'avoir accueilli le colloque, à Nadine Méouchy d'avoir organisé l'aspect logistique de notre séjour à Alep et à Damas, à Salam al-Kawakibi de son soutien organisationnel généreux à Alep. Nos chaleureux remerciements vont aussi au père Emile, notre hôte à Saint Assia, à Nuri Iskander qui a organisé nos concerts et aux musiciens et amis d'Alep qui nous ont rejoints. Le Groupe d'étude remercia chaleureusement à l'unanimité Scheherazade Hassan pour l'organisation d'un colloque qui nous permit d'expérimenter des dimensions importantes de la musique et de la culture dans le monde arabe. Nous espérons que les documents de la réunion seront publiés sous diverses formes en français, en anglais et en arabe. Une sélection des exposés présentés au congrès sera publiée dans le Bulletin d'Etudes Orientales, l'excellente revue de l'IFEAD et il est aussi question d'une publication de l'ensemble des communications dans un volume séparé. 